\title{
Role of Retrograde Intrarenal Surgery in Management of Renal Stones: 3 Years Experience
}

\author{
Yadav Rajinder*, Puri Anurag and Gupta Pravesh \\ Department of Urology, Fortis Hospital, Delhi, India
}

*Corresponding author: Yadav Rajinder, Department of Urology, Fortis Hospital, Delhi, India.

Received Date: June 03, 2019

Published Date: June 25, 2019

\begin{abstract}
Introduction and Objective: Retrograde Intrarenal Surgery (RIRS)is considered as a minimally invasive procedure for management of renal stones with minimal morbidity. Our objective is to demonstrate its effectiveness in management of not just small, but even large, multiple and staghorn stones.

Methods: A prospective study was done of 274 patients who presented to us with renal and upper ureteric stones and were managed with RIRS Preoperatively, stone size and laterality were assessed on NCCT KUB and X-ray KUB. Intraoperative parameters assessed were operative time, need for ureteric dilatation and intraoperative complications. Post operatively, check X-ray KUB/USG KUB was done before stent removal. Patients were followed up in outpatient department and telephonically.

Results: Out of 274 patients, 185 patients were males and 89 were females. 83 patients had single stone and 191 patients had multiple stones. 25 patients were pre stented in view of septicemia or renal impairment. 47 patients had renal impairment at the time of presentation, which improved in all patients and returned to normal value in 36 patients. 85 patients underwent bilateral RIRS and 189 underwent unilateral RIRS. 68 patients had stone size $<1 \mathrm{~cm}$., 99 patients had $1-2 \mathrm{~cm}$. and 107 patients had more than $2 \mathrm{~cm}$ sized stones. 6 patients had residual stones out of which, 3 patients underwent URS, 2 patients underwent RIRS and 1 patient underwent ESWL.
\end{abstract}

Conclusion: RIRS is feasible in large stone burden like partial and complete staghorn stones with minimal morbidity. Our study demonstrates its effectiveness in large stone burden(size $>2 \mathrm{~cm}$.), with additional procedure required in $<3 \%$ patients.

\section{Introduction}

The incidence of urinary stone disease is increasing all over the world due to environmental conditions in association with improving health services and diagnostic modalities [1,2]. The management of renal stones has evolved due to technological advancements in last few decades from open to percutaneous to very minimally invasive procedures. The success of these minimally invasive modalities has made open surgery for renal stone disease rare. PCNL (percutaneous nephrolithotomy) became the procedure of choice after its first description in 1976 for management of large burden renal stones and as a treatment option for small renal calculi [3]. Although PCNL had a good stone clearance rate, it is associated with a potential morbidity of bleeding which may need angioembolisation (0.6-1.4\%) Michel MS, et al. [4] and so has limitations in patients with bleeding diathesis. Also, PCNL is technically more demanding and is a morbid procedure associated with longer hospitalization, postoperative pain, longer bed rest, and longer time to return to work etc. With rapid advances in technology, miniaturization of endoscopes, improvement in fiberoptic technology, availability of holmium: YAG laser and other ancilliary instruments have rendered RIRS (Retrograde Intrarenal Surgery) a better opportunity for management of renal and ureteric stones. Also, RIRS is associated with low complication rate, minimal morbidity and early return to work.

We started flexible ureteroscopy in 2003 with the availability of 30 Watts Holmium laser. Initially we used to do diagnostic ureteroscopy and used to remove small stones left over after fragmentation by ESWL and PCNL and broken stents. With the availability of first flexible ureteroscope we could perform only 30 cases successfully. It was not possible to buy another flexible ureteroscope as it was costly. We borrowed the flexible laryngoscope and performed another 25 cases of removal of small stones from ureter and kidney. Both the scopes were from stortz. With the 
availability of baskets and flexible ureteroscope we performed RIRS in another 100 cases of upper ureteric and renal stones of up to 2 $\mathrm{cm}$. size. More than $2 \mathrm{~cm}$. size renal stones were treated by PCNL till 2013. With rapid advances in technology, miniaturization of endoscope and video endoscope and improvement in fiber-optic technology, availability of Holmium: YAG laser and other ancillary instruments has rendered RIRS (Retrograde intrarenal surgery) a better opportunity for management of renal and ureteric stones. Also, RIRS is associated with low complication rate, minimal morbidity and early return to work. At present RIRS is limited to patients where PCNL/ESWL are contraindicated because of presence of bleeding diathesis, patients with morbid obesity, malrotated kidney, malpositioned kidney and stone size up to 2 $\mathrm{cm}$ [5]. We have evaluated the feasibility and efficacy of RIRS for management of stones including those of stone size $>2 \mathrm{~cm}$ (including partial and complete staghorn). Staged RIRS is performed for patients with large stone burden (partial and complete staghorn stones) as an alternative to PCNL [6].

\section{Materials and Methods}

A prospective study was done from August 2013 to June 2016. 274 patients with renal and upper ureteric stones including stone size $>2 \mathrm{~cm}$ to multiple, bilateral including even partial and staghorn stones underwent RIRS at our institution. RIRS was considered the first choice for management of renal stones coming to our hospital irrespective of stone size. Patients were pre-informed about staged procedure if they had bilateral large renal stones. Preoperatively, Stone size and laterality were assessed on NCCT KUB, X-ray KUB films or CT urography. All the patients were investigated for comorbidity. All the patients had urine culture and sensitivity done before the procedure and RIRS was carried out only after urine culture was sterile. Most of the patients were admitted on the same day in the morning and those who were suspected to have infection or obstructed system were pre-stented and treated for a week with appropriate antibiotics to clear the infection and improve renal function in those cases where renal functions were deranged. Almost all patients were operated under general anesthesia except for few cases who were not fit for GA, were done under spinal anesthesia. We did not routinely pre stent the patient. Cystoscopy was performed in all patients to rule out any urethral obstruction or bladder abnormality and to assess the compliance of ureteric orifices. We used video-endoscopes and flexible ureteroscope from Storz and Olympus with double deflection.

All the patients were ureteroscoped by semi-rigid ureteroscope of size 7/8.5 Fr. and guidewire was inserted into the kidney and the ureteroscope was passed up to the renal pelvis. There was no need to dilate ureteric orifice in patients in whom ureteroscope could be passed till renal pelvis and 14/12 Fr. was easily negotiated up to PUJ without any difficulty. We used new ureteroscopic sheath in all patients except in pre-stented patients, where old sheaths were reused. Those patients in whom ureteroscope could not be negotiated, dilatation of orifice was done with balloon dilator. Few of the patients had stricture in ureter, which did not allow urteroscopic dilatation and underwent balloon dilatation. During these procedures, if the patient had pyonephrosis or turbid infected urine, we did not proceed further, and patient was left with the stent and RIRS was done at a second stage. Double guide wire was rarely used. All these procedures were carried out under C-arm guidance.

RGP was not done in most of the cases except in few where the calyces were in awkward position, just to guide the ureteroscope into a particular calyx. Sometimes to access a stone in a difficult calyx, the table was tilted towards right or left depending on the side of the stone or by placing a sandbag under the renal angle. In 9 cases where access sheath could not be negotiated, flexible ureteroscope was guided over flexible biwire into the renal pelvis. We did not reposition the stones from calyces in most cases (except in 4 cases). If calyx was not negotiable, we divided the infundibulum, diverticular or calyceal neck with laser wherever needed, particularly in lower calyx to fragment the stones. We used 200-micron laser fiber in lower calyces and middle calyx stone and 365 microns in upper calyx and pelvic stones. Our energy setting was 0.2 Joules and 10 Hertz. We used painting and popcorn effect in all patients to fragment the stones. We did not used drilling technique. By painting technique, we powdered the stone by keeping the laser fiber 1-2 mm. away from the stone. In most of the cases painting was started from one of the margins and continued on the margins only. At the end of fragmentation, the stone was fragmented by popcorn effect where laser beam was focused in the center of the calyx and fragments flew like popcorns coming in contact with laser and get hit by laser fiber to become tiny fragments. Fragments were not removed except for taking few pieces for chemical analysis.

The stones were observed under C-arm and larger fragments were fragmented if visible. The fragments were basketed by tipless basket or by engage basket for chemical analysis. All the patients were stented after passing a guidewire through the sheath and the sheath was withdrawn under ureteroscopic guidance to see any injury to ureter. The stents were inserted over the guidewire into the collecting system without Ureteroscope and cystoscope by pusher under C-arm guidance. Patient was catheterized for next 24 hours. Most of the patients were discharged after 24 hours and allowed to resume normal work after 2-3 days. All the patients were advised to come for follow-up after 1 week to see the progress. They were advised to get X-ray KUB done after 3 weeks prior to stent removal. If any fragments of stone were found in kidney or ureter, they were relooked and removed during stent removal.

\section{Results}

Our case series has the largest study populations in adults published in literature until now. We had in total 274 patients, 185 patients being males and 89 females. 83 patients had single stone, 96patients had multiple stones, 54 patients had partial staghorn stone and 16 patients had staghorn stone. According to stone size 68 patients had $<1 \mathrm{~cm}$ stone size, 99 patients had stone size $1-2 \mathrm{~cm}$ and 107 patients had $>2 \mathrm{~cm}$ stone size. 87 patients had unilateral renal stones, 85 patients had bilateral renal stones, 77 patients had renal with ureteric stones and 25 patients had upper ureteric stones (FIgure 1\&2). 


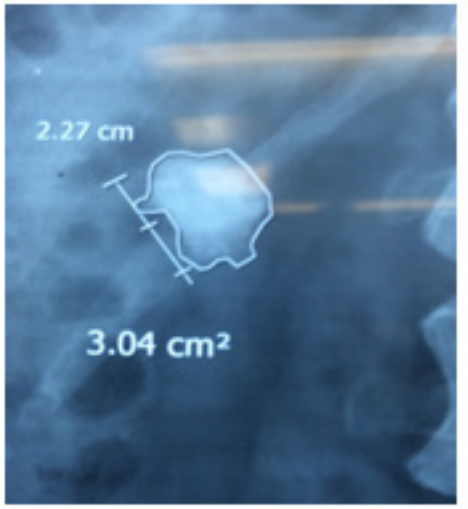

Before RIRS
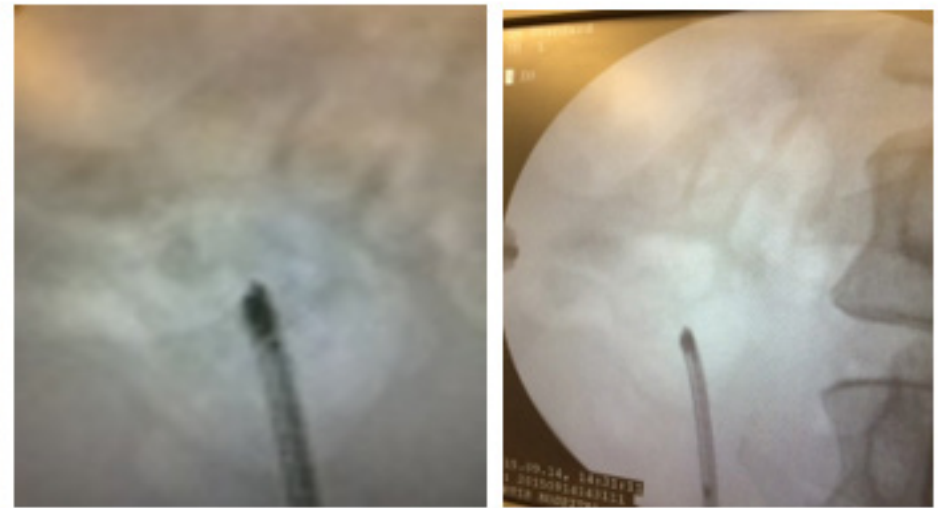

During procedure

Figure 1: Before and after images for partial staghorn stone.

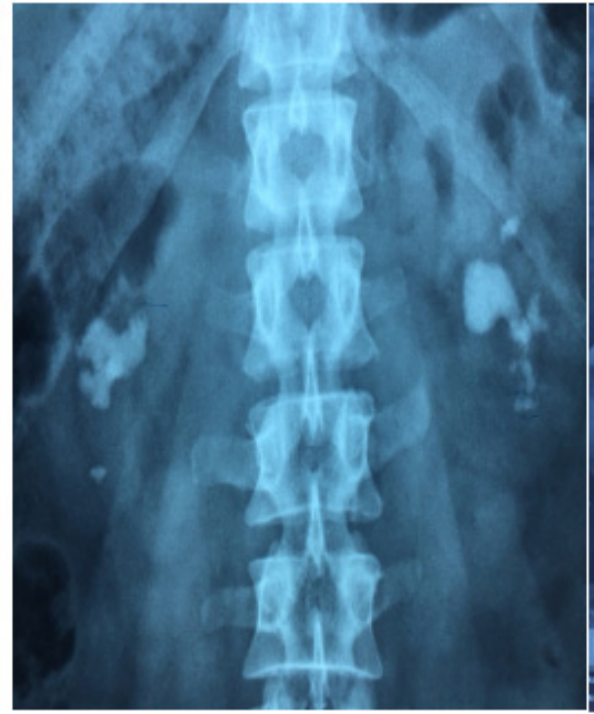

Before RIRS

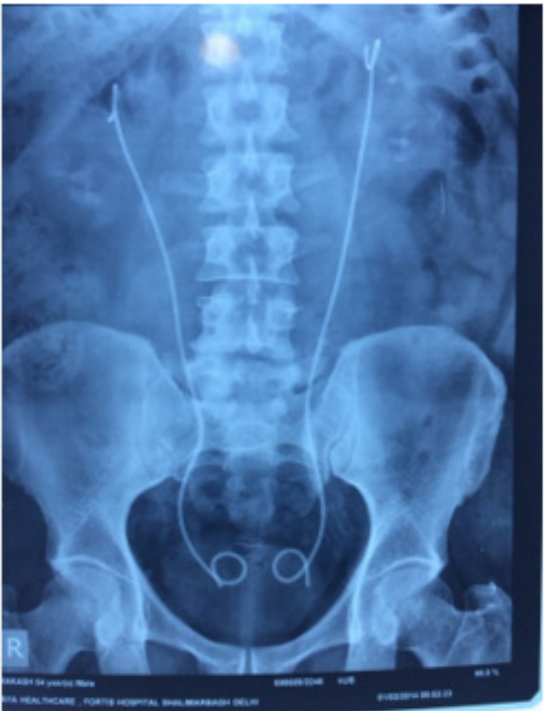

After $1^{\text {st }}$ stage RIRS

Figure 2: Before and after images for staghorn stones.

85 patients underwent B/L RIRS and 189 underwent U/L RIRS. 25 patients were pre stented in view of septicemia or renal impairment out of 274 patients. All 35 patients (who were prestented) underwent RIRS after 1 week of stenting when their general condition was stabilized. 47 patients had renal impairment at the time of presentation, which improved in all patients and returned to normal value in 36 patients. Operative time for RIRS in our patients ranged from 35 minutes - 160 minutes and average time was 85 minutes. 234 patients required no dilatation i.e. after passing ureteroscope access sheath passed easily over the guide wire. 25 patients had narrowing at VUJ (Vesicoureteric junction) and 15 patients had narrowing at multiple sites in ureter which required balloon dilatation. 4 patients were stented in view of failed dilatation and procedure was staged.

Post operatively 14 patients had urinary tract infection and 7 patients had hematuria which were managed conservatively.
One patient had ureteric perforation intraoperatively (during dilatation). RIRS was completed in same sitting in this patient after passing access sheath over guidewire and retrograde double J stent was placed at the end of the procedure. None of our patient had ureteric stricture even on long term follow up. Hospital stay ranges from 12 hrs - 2 days and average stay was 28 hrs. Post operatively patients had mild pain which were managed with analgesics. All patients returned to work within 3 days of RIRS. 38 (appr. 14\%) out of 274 patients had stent related symptoms (dysuria, flank pain during micturition etc.) which were settled with medications. All patients were evaluated with X-ray KUB (for radiopaque stones) and Ultrasound KUB (for radiolucent stones) before double J stent removal. Out of 274 patients, 268 patients (96\%) had complete clearance of stones in first sitting, 6 patients had residual stones out of which, 3 patients underwent URS, 2 patients underwent RIRS and 1 patient underwent ESWL. All the 6 patients were stone free after ancillary procedure. 


\section{Discussion}

Before the development of modern flexible scopes, PCNL was the only option to treat large upper ureteric and renal stones, although it had its own limitations being better for low volume disease, was associated with more complications mainly connected to longer procedure time, radiation exposure and hospital stay. In 1983, Huffman and associates first reported the use of ureteroscopy to treat renal pelvic stone [7].

Various studies have been done in the past where efficacy of RIRS has been found to be comparable to PCNL for stone size up to $2 \mathrm{~cm}$ [8-11]. Very few studies have been done where RIRS was done for stone size $2-4 \mathrm{~cm}$ and no study has been done to the best of our knowledge where RIRS has been employed in stag horn and multiple stones [12-13]. Prabhakar M, et al. [12], have recently done RIRS for stone size 1.6 to $3.5 \mathrm{~cm}$ (average size $2.5 \mathrm{~cm}$ ) on 30 patients and achieved a stone free rate of $86.6 \%$ in first sitting and $100 \%$ in second sitting. In a study by Haffron J, et al. [14], 14 patients were treated with combined RIRS and SWL (Shock wave lithotripsy) where they were either unfit for PCNL or refused PCNL. The calculated stone surface area was $847 \mathrm{~mm} 2$ (58mm2 $1850 \mathrm{~mm} 2$ ). Only $14 \%$ of patients were stone free after first sitting, whereas overall stone free rate was 77\%. Ricchiuti DJ, et al. [15] carried out a retrospective analysis of 23 patients treated with RIRS, found an overall stone free rate of $74 \%$. They found that lower pole stones had a better stone free rate $(83 \%)$ as compared to stones located at other sites (74\%).

Sabnis RB, et al. [16] compared microperc versus RIRS for management of small renal calculi. There were 35 patients in each group. They concluded that although microperc was a safe and effective alternative to RIRS but was associated with higher requirement of DJ stenting. Ho CC, et al. [17] did a retrospective study and reviewed the data of 46 patients who underwent RIRS for lower pole to calyceal stones of $<2 \mathrm{~cm}$. They found stone free rates was significantly better in the group that underwent primary RIRS as compared to the group undergoing RIRS after failure of SWL. In our study RIRS was done as a procedure of choice for all kind of stones except in cases where stone was associated with hydronephrosis and poor renal function, or PCNL was the preferred option by the patient which was rare. Almost 98\% of patients came to our center for RIRS inspite of being advised for PCNL for large stones, staghorn stones and multiple stones at other centers, although we offered them all modalities including PCNL.

Stone free rate in our study was $96 \%$ in first sitting and $99.8 \%$ in second sitting. In one patient ESWL was done to clear the stone and another patient had three sittings. Most of the time second sitting was done during stent removal, removing fragments visible on X-ray KUB. We have been incising infundibular and narrow calyceal neck with laser in difficult situation to achieve better access to stone free rate. Only in one case we could not reach the stone in the lower calyx inspite of incision where ESWL was done. It is always advisable to paint the stone of any size rather than fragmenting, as fragments go to different calyces and it becomes tedious to break the stone in each calyx. We also take the anesthetist's help in minimizing the movement of the kidney by decreasing the tidal volume. By combining above mentioned techniques we have been able to achieve a better stone free rate with lesser complications as compared to other studies.

RIRS is replacing ESWL and PCNL in management of renal stones as first choice. RIRS is safe, efficacious, reproducible with minimal morbidity and faster recovery. RIRS is feasible in large stone burden like partial and complete staghorn with minimal morbidity. Our study demonstrates its effectiveness in large stone burden(size $>2 \mathrm{~cm}$.), with additional procedures required in $<3 \%$ patients.

\section{Conclusion}

A stone free rate of $96 \%$ was achieved in our study in first sitting for patients of renal stones treated by RIRS. Based on our encouraging results and as per reports in literature RIRS may be considered as a safe and effective procedure in all kinds of renal stones (small, big, bilateral and staghorn) with higher stone free rates, low morbidity and less auxiliary and re-treatment rates. Patients have to be informed about the possibility of staged procedure when RIRS is considered as treatment option to attain a higher stone free rate. At our center RIRS has already replaced other procedures of stone removal for all kinds of single, multiple and bilateral and staghorn stones with comparable stone free rate to PCNL with added advantage of lesser complications, shorter hospital stays and quicker resumption of work post-operatively.

\section{Acknowledgements}

None.

\section{Conflict of Interest}

No conflict of interest.

\section{References}

1. Romero V, Akpinar H, Assimos DG (2010) Kidney stones: A global picture of prevalence, incidence, and associated risk factors. Rev Urol 12(2-3): e86-e96.

2. Clark JY, Thompson IM, Optenberg SA (1995) Economic impact of urolithiasis in the United States. JUrol 154(6): 2020-2024.

3. Fernstrom I, Johansson B (1976) Percutaneous pyelolithotomy: A new extraction technique. Scand J Urol Nephrol 10(3): 257-259.

4. Michel MS, Trojan L, Rassweiler JJ (2007) Complications in percutaneous nephrolithotomy. Eur Urol 51(4): 899-906

5. Watterson JD, Girvan AR, Cook AJ, Beiko DT, Nott BK, et al. (2002) Safety and efficacy of holmium: YAG laser lithotripsy in patients with bleeding diasthesis. J Urol 168(2): 442-445.

6. Ricchiuti DJ, Smaldone MC, Jacobs BL, Smaldon AM, Jackman SV, et al. (2007) Staged retrograde endoscopic lithotripsy as alternative to PCNL in selected patients with large renal calculi. J Endourol 21: 1421-1424.

7. Huffman JL, Bagley DH, Lyon ES (1983) Extending cystoscopic techniques into the ureter and renal pelvis: Experience with ureteroscopy and pyeloscopy. JAMA 250015: 2002-2005.

8. Chung BI, Aron M, Hegarty NJ, Desai MM (2008) Ureteroscopic versus percutaneous treatment for medium size $(1-2 \mathrm{~cm})$ renal calculi. J Endourol 22(2): 345-346. 
9. Sofer M, Watterson JD, Wollin TA, Nott L, Ravzi H, et al. (2002) Holmium YAG laser lithotripsy for upper urinary tract calculi in 598 patients. Urol 167(1): 31-34

10. Sabnis RB, Jagtap J, Mishra S, Desai M (2012) Treating renal calculi 1-2 $\mathrm{cm}$ in diameter with minipercutaneous or retrograde intrarenal surgery: A prospective comparative study. BJU Int 110(8 Pt B): E346-E349.

11. El Nahas AR, Ibrahim HM, Youssef RF, Sheir KZ (2012) Flexible ureterorenoscopy versus extracorporeal shock wave lithotripsy for treatment of lower pole stones of 10-20 mm. BJU Int 110(6): 898-902.

12. Prabhakar M (2010) Retrograde ureteroscopic intrarenal surgery for large (1.6-3.5 cm) upper ureteric/renal calculus. Indian J Urol 26(1): 46-49.

13. Giusti G, Maugeri O, Benneti A, Piccinelli A, Taverna G, et al. (2010) Retrograde Intrarenal Surgery (RIRS) in the treatment of renal stones between 2 and $4 \mathrm{~cm}$. J Urol 183: 700-701.
14. Hafron J, Fogarty JO, Hoenig DM (2005) Combined ureteroscopy and shockwave lithotripsy for large renal stone burden, an alternative to percutaneous nephrolithotomy? J Endourol 19(4): 464-468.

15. Ricchiuti DJ, Smaldone MC, Jacobs BI, Simaldone AM, Jackman SV, et al. (2007) Staged retrograde endoscopic lithotripsy as an alternative to PCNL in selected patients with large renal calculi. J Endourol 21(12): 142-144.

16. Sabnis RB, Ganesamoni R, Doshi A, Ganpula AP, Jagtap J, et al. (2013) Micropercutaneous nephrolithotomy (microperc) vs retrograde intrarenal surgery for the management of small renal calculi: a randomized controlled trial. BJU Int 112(3): 355-361.

17. Ho CC, Hafidzul J, Praveen S, Goh EH, Bong JJ, et al (2010) Retrograde intrarenal surgery for renal stones smaller than $2 \mathrm{~cm}$. Singapore Med J 51(6): 512-515. 